\title{
International Neuroblastoma Response Criteria
}

National Cancer Institute

\section{Source}

National Cancer Institute. International Neuroblastoma Response Criteria. NCI

Thesaurus. Code C102869.

A standard approach to aid in the stratification of neuroblastoma risk and disease evaluation. 